\title{
Considerations to Achieve Pulsatility for Left Ventricular Assist Devices through BLDC Motor by Open Loop Control System
}

\author{
Yenumula Vinod Kumar ${ }^{1}$, Dr. S.Venkateshwarlu ${ }^{2}$, Dr. Anil Kumar Puppala ${ }^{3}$ and Phani Kumar K.S.V ${ }^{4}$ \\ ${ }^{1}$ PG Scholar, CVR College of Engineering/EEE Department, Hyderabad, India. \\ Email: yenumulavinod123@gmail.com \\ ${ }^{2}$ Professor, CVR College of Engineering/EEE Department, Hyderabad, India. \\ Email: svip123@gmail.com \\ ${ }^{3}$ Professor, Geethanjali College of Engineering and Technology/EEE Department, Hyderabad, India. \\ Email: apuppala@gmail.com \\ ${ }^{4}$ Asst. Professor, CVR College of Engineering/EEE Department, Hyderabad, India. \\ Email: phani5016@gmail.com
}

\begin{abstract}
Ventricular Assist Devices (VADs) and Total Artificial Heart (TAH) are used to replace the diseased heart and play a major role in saving the lives of the people. Left Ventricular Assisting Devices are the mechanical circulatory devices which play a key role in assisting patients who are suffering from different heart problems. In earlier stages, pulsatile flow devices are used, and later they are switched to continuous-flow devices. Though these devices have several advantages they have reported several complications during working. To avoid these complications, several trails were being conducted. In this paper, the work is done on achieving the pulsatility in Ventricular Assisting Devices. The open-loop control system has been implemented which is used to get different speeds of the BLDC motor to achieve the pulsatile flow in the device. The results are compared to the earlier experiment results and are tabulated.
\end{abstract}

Index Terms: Left Ventricular assisting devices, pulsatile flow, BLDC motor control, percutaneous cables.

\section{INTRODUCTION}

In the past few years, cardiac diseases are the main reasons for the death of many people. Due to less availability of heart donors, many people who are suffering from heart disease who are waiting for the replacement of their hearts with normal hearts are dying. To overcome this problem, there is a need for developing a device that will support the circulatory system of the body. There is an ongoing development of these circulatory devices and heart replacement devices from the past 62 years [1]. The step towards the development of Mechanical Circulatory Devices (MCD) [2] has been started in 1957 and the field of MCD is the first introduced in 1964 [3-4]. From the past few decades, there are many deaths recorded due to heart failure. This motivated many of the engineers to develop MCDs. Mechanical circulator support devices are used to help in pumping the blood from the heart to other parts of the body in case of heart failure. These devices are used in two different therapy systems which are called bridge to recovery and bridge to transplant. In the first strategy, the heart recovers due to the support of Ventricular Assist Devices, and in the second strategy it ensures the patients' survival until a donor or total artificial heart can be transplanted. In the inception of the heart program in 1964 investigations have been conducted to develop the Total Artificial Heart [5] and Ventricular assisting Devices that are implantable inside the body without causing any damage to the skin. To implant these devices in patients' body, they have to undergo many clinical trials that involve various designs and different optimizations. During this process, it is needed to design these devices in such a way that they must fit the body without any problems and the weight and dimensions of these devices must be considered properly to implant into the patients' body. To meet this requirement, the blood pump, electric motor, the power supply should be small and these electric motors and blood pumps must be designed in a size such that they can be easily fit into the human body and sufficiently durable to support the patient for a longer duration and have a good performance efficiency. In the previous cases, many of the experiments were conducted on continuous flow systems. Due to many advantages of these systems such as ease of control and smaller in size they have been implemented in the body as a mechanical support system. Despite their advantages, continuous-flow devices have been reported several complications in providing a pulsatile flow of blood which resulted in several problems such as frequent hospitalization, etc., To overcome this problem, many of the experiments have been recently conducted to achieve the pulsatile nature which is the natural working of the heart. It is possible to achieve pulsatile flow by changing the speed of the motor \& pump at the rate of native heart rate [6-7]. To get the different speed levels of the motor, there is a need to control the speed using some controlling action. In the present paper, an open-loop control system to control the speed of the BLDC motor is being used.

\section{LiteratuRE REVIEW}

Gregory K. MacLean, Peter A. Aiken, William A. Adams, Tofy Mussivand (1994) conducted a study between $\mathrm{Ni} / \mathrm{Cd}$ and lithium cells which are used for implantable medical devices. In this process, they have used 2 different 
rectangular prismatic $\mathrm{Ni} / \mathrm{Cd}$ cells and 5 different rechargeable lithium cells and determined at $37^{\circ} \mathrm{c}$ temperature [8].

E. Okamoto, K. Tomoda, K. Yamamoto (1994) talked about a highly efficient, totally implantable assisting pump device, and also compact which has a motor-driven assisting pump. The volume of the pump which they have is used is $350 \mathrm{ml}$, and its controller is $210 \mathrm{ml}$. The outflow of the pump is $5.8 \mathrm{~L} / \mathrm{min}$ which is obtained against a mean after-load of $100 \mathrm{~mm} \mathrm{Hg}$ [9].

P J Ayre, N H Lovell, J C Woodard (2003) studied the effects of pulsatile and non-pulsatile flows using in vitro mock loops, and acute $(\mathrm{N}=3)$ and chronic $(\mathrm{N}=6)$ ovine experiments. An estimation of average flow in its algorithm was derived from the RMS pump impeller speed and RMS input power using the non-pulsatile and pulsatile mock loops. By using this algorithm, it has been estimated the flow in a rotary blood pump without implanting additional invasive sensors [10].

Allen Cheng, Christine A. Williamitis, and Mark S. Slaughter (2014) compared between continuous flow and pulsatile flow devices are considered and they have explained the advantages and disadvantages of different devices [11].

A. K. Puppala, V. Sonnati, and S. Gangapuram suggested a few materials for additive manufacturing. They thought that additive manufacturing would increase the performance of VADs. They reviewed a few materials like Ti-6A1-4V, Co-Cr-Mo, Co-Ni-Cr-Mo which would be suitable for applications related to the heart [12].

\section{Working OF LEFT VENTRICULAR ASSIST DEVICES}

Before knowing the working of LVADs the functioning of the heart is described. The heart is a muscular organ which is used to pump the blood continuously throughout the body. The heart is a muscular organ that consists of four chambers; they are, namely, the right and the left atrium and the right and the left ventricle. These chambers of the heart work together by contracting and relaxing alternatively in pumping blood throughout the body. The below figure describes the structure and functioning of the heart.

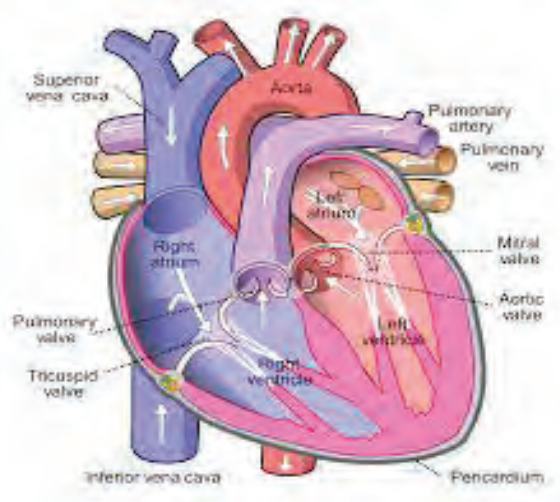

Figure 1. Structure and functioning of heart
After knowing the functioning of the heart, the working of Ventricular Assisting Devices which are used to assist the heart failure is presented. These devices are placed inside the body and are connected between the apex of the left ventricle to the aorta which is considered the main artery of the heart which is used to carry the oxygenated blood to the entire body. Blood is continuously sent from the left Ventricular Assisting chamber via the apical inflow canal and propelled through a pumping house where the magnetic field generation by a rotary pump transmits blood through the outflow graft to the ascending aorta. A percutaneously tunnelled driveline connects the external power source or the systems controller to the pump. The figure shown below indicates how the left Ventricular Assisting Devices (VADs) are connected to the human body.

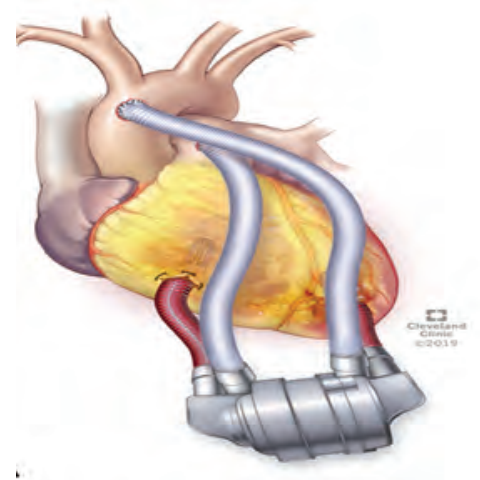

Figure 2. The Left Ventricular Assist Device connected to heart with controller and battery assembly

The system controller is the main component that is placed outside the body has both manual and fixed settings that modulate the speed of the pump, provided alarms where there is any malfunction in the device for future analysis. These Ventricular Assisting Devices deliver 4 to $8 \mathrm{~L}$ per minute of blood at different speed levels. The VAD has a centrifugal, pulsatile flow, implantable rotary pump currently in clinical practice. This Ventricular Assisting Device operates via a hydro-magnetically levitated rotor without mechanical bearings and can deliver up to 4.5 to $5 \mathrm{~L} / \mathrm{min}$ of blood through the heart.

\section{DESIGN OF LVADS}

\section{A. Internal Structure of the LVAD}

The design of the LVAD [13] is considered below. The design of the LVAD must be done in such a way that it must be small in size and be able to fit inside the body. The design of the LVAD requires different components like a pump which consists of motor, inlet and outlet valves, impeller unit, and housing which is used as a covering for these parts of the pump. Before designing the LVAD, there is a need to know how the LVAD internal structure looks like. 


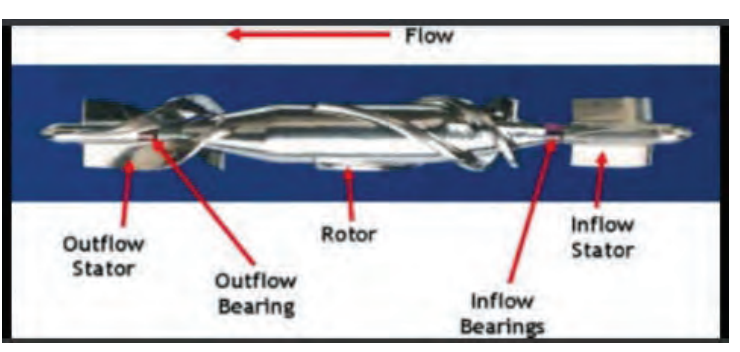

Figure 3. Internal structure of LVAD pump

The above diagram represents how the pump of the LVAD looks internally. It consists of an inflow stator from where blood enters the pump from the inlet valve. It is connected to the rotor of the motor with a shaft and bearings for free rotation of the inflow stator. This will rotate when the rotor starts rotating and it is used to drive the flow of blood inside the pump. After this inlet stator, there is a rotor that is known as the impeller unit of the pump which consists of a blade on it. This impeller unit is used in this system because the impeller is a rotating part of the pump which is able to convert mechanical energy given by the motor into power of the pump output which is fluid output by rotation. After the rotor, it consists of an outflow stator which is also connected to the rotor with the help of outflow bearings. This will allow blood to flow smoothly from the pump to the outlet valve. The Figure 4 represents the entire internal structure of the LVAD which has the inlet and the outlet valves. The inlet valve is connected at the apex of the left ventricle from where the oxygenated blood will enter from the right ventricle. From this inlet valve, blood enters the pump of the device.

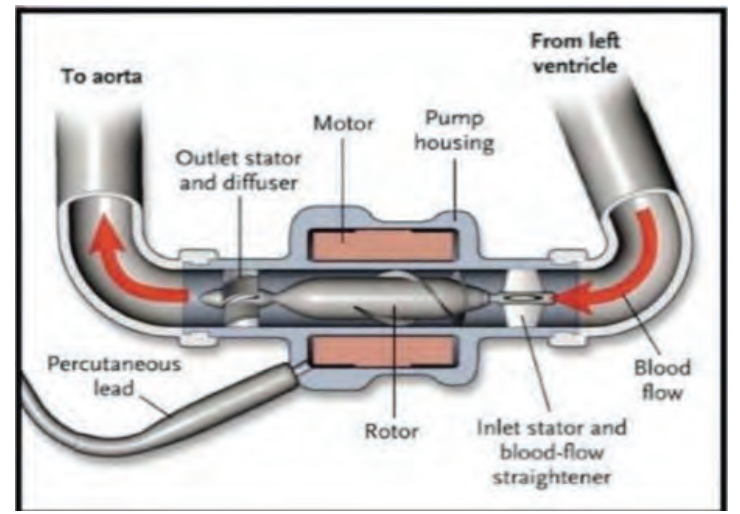

Figure 4. Internal structure of the LVAD with the inlet and the outlet valves

The outlet valve is connected to the Aorta which is considered the main artery of the heart which will deliver the oxygenated blood to the entire body. From the output side of the pump, blood will enter the outlet valve and from there, blood will enter the aorta. This outlet valve is connected to the pump with the help of strain relief. This strain relief is also referred to as bend relief that is required because it gives mechanical strength to the valve when the load is applied to the valve. By considering this requirement, a small device, which is totally implanted inside the body [14] has been designed.

\section{B. The BLDC Motor}

In the above section, the internal structure of the LVAD is described. From the above discussion, it is clear that the pump which is present in the LVAD is the key component in which the motor is present and due which blood is allowed to flow from the LVAD to the entire body. The motor is an important part of the pump which should be very small in size and can be fit inside the pump. During this study, there is a use of the BLDC motor. The construction of the BLDC motor is as if the construction of the PMSM. The invention of the brushed DC motor was done in the 19th century. These DC motors consist of brushes and the commutator assembly. Due to these brushes, there is friction along with the rotating commutator segments which will lead to power loss and this will make the motor as low power motor. Another disadvantage of a brushed DC motor is the resistance of the sliding brush contact which will lead to a voltage drop in the motor circuit which is also known as brush drop that will consume the energy. So, considering the above disadvantages of the brushed DC motor, the alternate motor is made which is known as brushless DC motor. These BLDC motors came into existence around the 1970s.

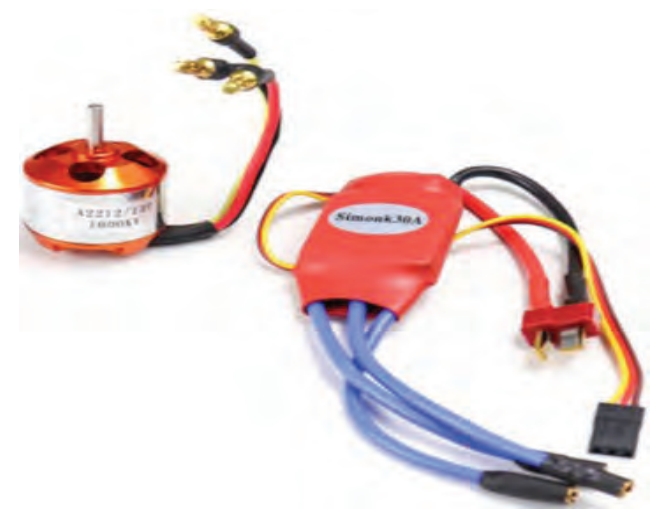

Figure 5. The BLDC motor with Electronic Speed Controller

The above figure represents the BLDC motor which is considered for testing purposes in this study. The BLDC motor is synchronous motors that are powered by DC electricity with the help of an inverter circuit which produces AC electricity as output which is used to drive each phase of the motor as a closed-loop controller. A Brushless DC motor has a commutation process done using a microcontroller. These motors use a hall-effect sensor which is used to determine the position of the rotor shaft. To rotate the BLDC motor, the stator windings must be energized in a particular sequence. The commutation circuit which is present inside the motor must know the position of the rotor to energize the correct winding. To do this, the BLDC motor consists of a hall-effect sensor. In a threephase motor, there are three hall sensors present in it. When there is a moment of rotor magnetic poles near the hall sensor, it will produce a signal indicating there is a pole 
passing near the sensor. Based on these combinations, the determination of the position of the rotor and according to the position, the energized stator windings can be done.

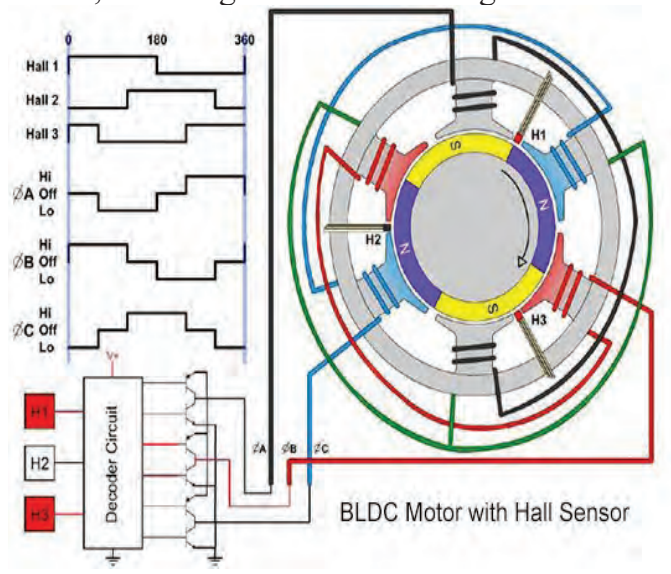

Figure 6. BLDC motor with hall sensors

This is the basic working of a BLDC motor using a halleffect sensor. In the VADs, the most commonly used motor is the BLDC motor. This is because the BLDC motor has several advantages over many other motors and the requirements for the VADs are met by the BLDC motor. Generally, the power ratings of TAH and VADs are in the range of $8 \mathrm{~W}$ to $20 \mathrm{~W}$ and the duration of these devices is around 2 years [15]. In this study, for testing of motor, A2212/1000kV motor is used which has $7 \mathrm{~V}$ to $12 \mathrm{~V}$ input voltage and $0.5 \mathrm{~A}$ of no-load current. From the study of the functioning of the heart, it is clear that the heart must pump around 5 to 6 litres of blood per minute. So, this BLDC motor should be able to pump 5litres of blood in one minute which can be done by adjusting the speed of the motor. The pulsatile flow of blood can be achieved maintaining constant torque at variable speeds with the motor-pump set which is used in TAH and VAD. This is another reason to use the BLDC motor in these devices. In a brushed DC motor, it consists of brushes for delivering current. If this motor is used for a longer period of time the brushes will worn out and they will produce sparking which is not allowed. So, in order to overcome this problem, the BLDC motor is used which doesn't have brushes which will remove the sparking effect. The BLDC motor also has many advantages like high torque to weight ratio, increase in reliability, it has less noise compared to the brushed DC motor, and a longer lifetime. So, the BLDC motor is an ideal choice for TAHs and VADs.

\section{Experimental Setup Controlling the Speed of the BLDC Motor by Potentiometer}

After studying the internal structure of the LVAD, it is clear that it uses the motor inside the pump which is used for the flow of blood through the heart. Before implanting the BLDC, motor inside the pump for designing the LVAD, the speed of the motor must be controlled and verified that when the power supply is given to the motor, it must rotate at the desired speed. The speed of this motor can be tested using different components like Arduino nano, potentiometer, and proximity sensor.

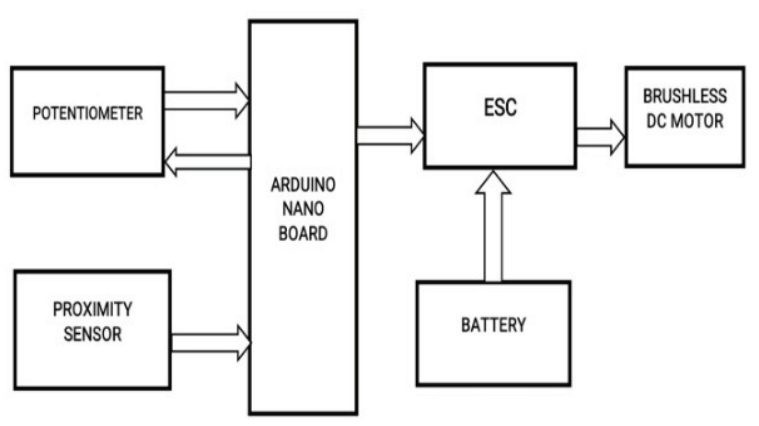

Figure 7. Block diagram of the BLDC motor with potentiometer and proximity sensor.

The above figure represents the block diagram of the BLDC motor connection to find the speed of the motor. In this first phase, the motor is controlled manually using a potentiometer which has potentiometer is a three-terminal device and it is generally a voltage divider to measure voltage or electric potential. These instruments are commonly used to control electrical devices mainly to control the speed of the motors. It consists of a sliding contact, when it is rotated, it will increase or decrease the resistance value through which the voltage can be limited, and ultimately, the speed of the motor can also be controlled. Another device that is taken into consideration is the proximity sensor. A proximity sensor is a device that is used to sense the presence of objects which are closer to it without any physical contact between the object and the sensor. A proximity sensor will emit an electromagnetic field that looks for changes in the field and returns that change to it. This sensor is used to detect the obstacle when it enters the field. The proximity sensor returns the signals in the form of $0 \mathrm{~s}$ and $1 \mathrm{~s}$. If there is no obstacle detected, it will return the value as 0 and if there is any obstacle is detected, it will return the value as 1 . A battery is also shown in the block diagram. In this study, Lipo battery of rating $11.1 \mathrm{~V}$, $2200 \mathrm{mAh}$ is used to give power supply to the motor. The other component, it is being used i.e. Arduino nano which consists of a microcontroller to do operations using the nano-board. The program which is used to rotate the motor and is used to control the speed of the motor using a potentiometer is written and dumped in this Arduino Nano board.

\section{Algorithm for analysing speed of rotation:}

$>$ Set and initialize the variables such as proxy, s, Val, prev_state, current_state, count.

$>$ Initialize current_state is equal to proxy.

$>$ Check for the condition of current_state is not equal to prev_state.

$>$ If the condition is false again go back to STEP 2.

$>$ If the condition is true check where the obtained current_state is equal to one.

$>$ If the condition is false again go back to STEP 2. 
$>$ If the condition is true increment the count value by $1($ count $=$ count +1$)$.

$>$ After completing the loop initialize prev_state value is equal to current_state value.

The above algorithm describes how the code is written in the Arduino board to rotate the motor and also used to detect the speed of the motor. The BLDC motor is connected with the nano board with the help of Electronic Speed Control (ESC). The ESC is an electronic circuit that is used to control and regulate the speed of the motor. This will follow a speed reference signal which is used to energize the coils at particular instants of time. In the ESC, it consists of MOSFETS as a switch and if the BLDC motor has Hall sensor, it will send signals which represent the position of the rotor. In another case, if a BLDC motor without a hall sensor is used using back-emf, it will send the signals which represent the position of the rotor. According to the position of the rotor, the respective pair of switches will get closed and the remaining switches will be in off mode.

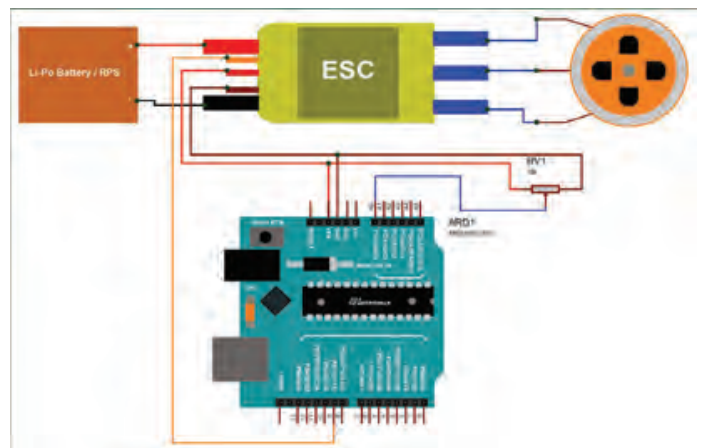

Figure 8. Experimental setup to find the speed of the BLDC motor using proximity sensor

The above figure represents the experimental setup to find the speed of the BLDC motor using the proximity sensor. When the power supply through the battery is given to the motor, it will start rotating. A small piece of paper or wire is attached at the tip of the motor which can be detected by the sensor. While the motor is rotating the piece of paper or wire also starts rotating. This is taken as an obstacle for the proximity sensor and whenever it detects the piece, it will send the signal and the value of count is increased. By using this count value which will appear on the serial monitor, the speed of the motor can be determined.

\section{5 \\ 161718192021222324252627282930}

Figure 9. Serial monitor output for speed control of motor

By considering the values in the serial monitor after $30 \mathrm{sec}$, the speed of the motor (SPEED = value we get on the serial monitor after $30 \mathrm{sec}$ ) can be calculated. By using a potentiometer, the speed of the BLDC motor can be varied and this can be done by varying the potentiometer nob manually.

\section{E. Open Loop Control System}

From the above, it is seen that how the speed of the BLDC motor can be controlled manually using a potentiometer and the speed of the motor can be determined the proximity sensor. In the above system, there is manual control of the motor is considered. But, when the motor is used in the design of the LVAD which is placed inside the body, the speed of the motor must be automatically controlled to achieve a pulsatile nature flow of blood. So, this is the reason the open-loop control system is considered in this study.

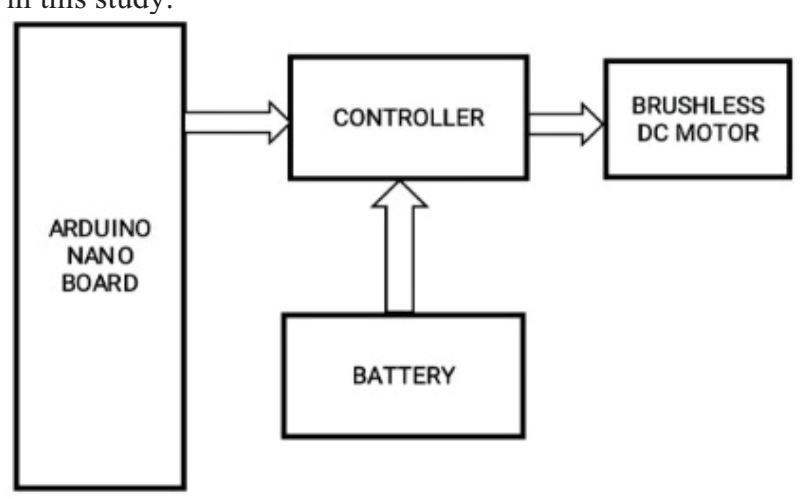

Figure 10. Block diagram for open loop control system

The above figure represents the block diagram for the automatic speed control of the motor using an open-loop control system. In this Arduino nano, a controller (ESC), the BLDC motors, a battery that powers the BLDC motor used.

\section{F. Algorithm for open loop control:}

$>$ Initialize the values of proxy, value, count and declare them as integers.

$>$ set proxy value as input parameter, the motor attached pin value, baud rate of serial monitor.

$>$ check whether the value of count is not equal to 2 .

$>$ If the condition is true the loop begins. The motor speeds will increase from 1200 to 1600 and the motors rotates at that speed for $0.2 \mathrm{sec}$.

$>$ In the next step the speed of motor will reduce from 1600 to 1400 and the motor will rotate at that speed for $0.15 \mathrm{sec}$.

$>$ Again, the motor speed is reduced from 1400 to 1300 and will rotate for $0.15 \mathrm{sec}$.

$>$ From that speed the speed will get further reduced to 1220 and the motor will rotate for $0.1 \mathrm{sec}$.

$>$ In the last step the motor will gain the starting speed which is 1200 and rotate for $0.4 \mathrm{sec}$.

$>$ After completing this loop, the count value is increased and again, it goes to STEP 3.

$>$ The loop will continue until the value count is equal to 2 . After this the loop will end and we will come out of the loop and the program will stop. 


\section{0}

\section{0}

Figure 11. The output values obtained from serial monitor for an open loop system

From the above figure, it is observed in the serial monitor that the speed values of the motor which is varying in certain intervals of the time.

So, this is how the speed of the motor can be varied which is used to design the Ventricular Assisting Devices that are used to pump the blood in a pulsatile manner which represents the normal functioning of the heart. The speed of the motor is varied in an open-loop control system to achieve the pulsatile flow of blood. Due to these variations in the speed of the motor the blood which is pumped from the LVAD will resemble the normal heart pumping.

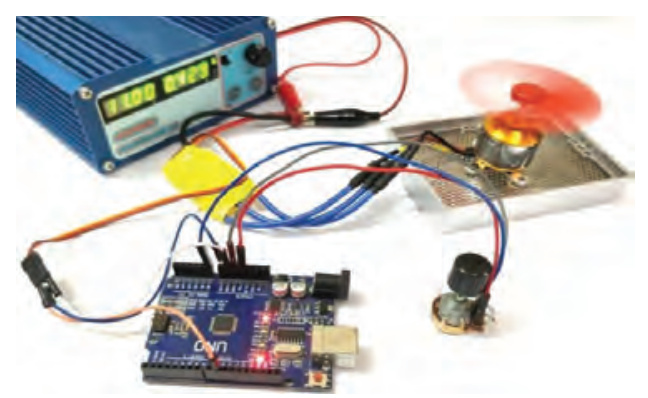

Figure.12. Experimental setup for an open loop control system

The above diagram represents the experimental setup of an open-loop control system. By this setup, the required code is written and dumped in the nano board and the speed of the motor at different intervals of the time is recorded and are verified with the previous results.

\section{BATteries USED AND PoWer TRANSFerred For Ventricular AsSisting DeVices}

The rechargeable battery pack is used for Ventricular Assisting Devices. The batteries which give power supply to the LVAD must be easy and effective in operation. The use of rechargeable lithium cells in implantable medical devices may yield batteries that are smaller and lighter than those of $\mathrm{Ni} / \mathrm{Cd}$ batteries, however, $\mathrm{Ni} / \mathrm{Cd}$ cells are less preferred than lithium batteries because of its high discharge cycle and life cycle. Various batteries were considered for supplying power to Ventricular Assisting Devices (VADs). In the recent days, Lithium-ion batteries have more advantages like low discharge rate, high power supply, and less weight when compared to different batteries. So, in the recent studies in giving power supply to the LVAD Lithium-ion batteries are used. Two 14V lithium-ion battery packs were used to give power supply. These battery packs are connected to the controller and it will supply the power to the motor which is implanted inside the body.

To give power to the motor, there is a need to develop a connection between battery packs and the motor. Here, there are the percutaneous cables [16] which are used to transmit the electrical energy from a battery source which is located outside the body to the motor which is present inside the body. These types of cables are passed through the skin and which will give power to the implanted motor.

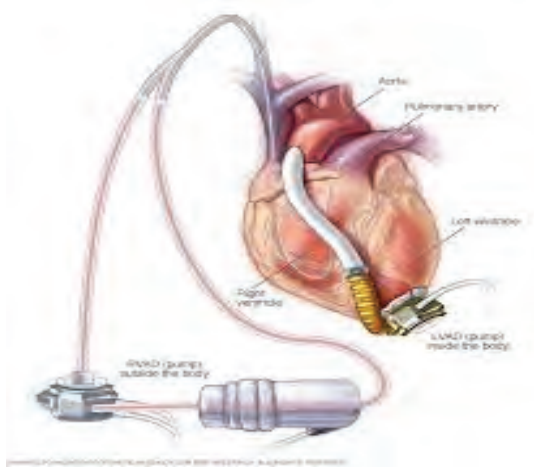

Figure 13. Percutaneous cable type transmission

\section{EXPERIMENTAL RESULTS}

This paper implemented an open-loop control system to achieve the pulsatile speed in the BLDC motor (hall sensorbased) which is placed inside the pump which is used to design the LVAD.

The experimental results for the open-loop control system are obtained for different levels of speed at different intervals of time and are tabulated as below. TABLE I.

DiFFERENT LEVELS OF SPEED AT DIFFERENT INTERVALS OF TIME

\begin{tabular}{|c|c|c|}
\hline S. No & Speed (rpm) & Time (sec) \\
\hline 1 & 1200 & $0+$ \\
\hline 2 & 1600 & 0.2 \\
\hline 3 & 1400 & 0.35 \\
\hline 4 & 1300 & 0.5 \\
\hline 5 & 1220 & 0.6 \\
\hline 6 & 1200 & 0.7 \\
\hline 7 & 1200 & 0.1 \\
\hline
\end{tabular}

These levels of speed are considered from the previous results and are used in the present work to get the pulsatile flow of blood. This work is done by using Arduino nano through which the open-loop operation is done and made to rotate the motor at different levels of speed. These levels of speed of the motor which are varied during particular intervals of time are plotted in the graph and the output of the graph obtained is shown below.

The peak in the graph is observed because initially, the heart is in a contraction state in which the blood is pumped from the left ventricle to the aorta with some pressure. Hence, the open-loop system is set up for initial speed to be high. After that, the heart will go into a relaxation state in which the heart is slowly relaxed. Due to this, speed is slowly reduced and made constant. 


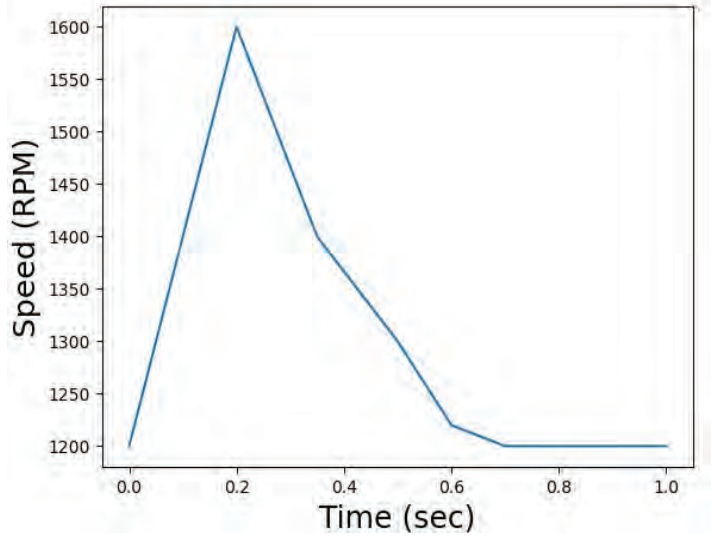

Figure 14. Pulsatile speed obtained with the BLDC motor using open loop control system

\section{CONCLUSIONS}

The weight, dimensions, and simplicity in control of the speed of the BLDC motor offer a practical solution for VAD. Control of the speed of the motor can be achieved by using different methods. While hall sensor-based operation for speed control is suitable for the devices. In the present paper, an open-loop control system is used to achieve the various levels of speed of the motor at different intervals of time. By using this open-loop system, pulsatile flow of blood is obtained which resembles the normal functioning of the heart.

Although this open-loop system is easy to implement, it has certain disadvantages. If there is no disturbance in the system, this open-loop system will work normally. But, if there are any disturbances in the system, it will affect the speed of the motor. Ripples will occur due to the disturbances present in the system. These ripples may lead to an uneven flow of blood inside the body. So, for the future, a closed-loop control system can be used to overcome this type of problem. The speed of the motor is fed back to the controller and this controller will compare the fed back speed and the original speed in which the motor has to rotate and reduce the error in the speed signal. Due to this, the speed of the motor can be controlled at the desired speed.

\section{REFERENCES}

[1] Benedikt A. Pelletier, Yvonne M. Blaszczyk, Patrick Carstens, Gonzalo Alvarez, Felix Lamping, Marco Laumen, Thomas Finocchiaro, and Ulrich Steinseifer on "Novel Optical Position Sensing for Miniaturized Applications and Validation in a Total Artificial Heart" IEEE transaction on biomedical engineering, VOL. 63, NO. 3, MARCH 2016.

[2] James F. Antaki, Richard K. Wampler, Kormos. M. "Mechanical circulatory support. A companion to Heart disease". Elsevier Saunders 1995.

[3] Leslie mertz on "From artificial kidneys to artificial heart and beyond";14 IEEE Pulse May 2012.
[4] Sean Pinney .P, on "Left Ventricular Assist Devices: The Adolescence of a Disruptive Technology" Journal of Cardiac Failure Vol. 21 No. 102015.

[5] T. Vakhtang, S. Phillips "Update in Artificial Heart Technology: Are We there yet?" Artificial Organs Vol 40., No.12,2016.

[6] Francis D. Pagani on "The quest towards the Holy Grail of mechanical circulatory support"; The Journal of Thoracic and Cardiovascular Surgery, September2015.

[7] R. Basanth, Anil K. Puppala "Considerations for Introducing Pulsatility in Continuous flow Ventricular Assist Devices by Modulation of Motor Speed," IEEE International Conference on Innovative Technologies in Engineering 2018 (ICITE OU).

[8] Gregory K. MacLean, Peter A. Aiken, William A. Adams, Tofy Mussivand "Comparison of rechargeable lithium and nickel/cadmium battery cells for mechanical circulatory support devices". ASAIO J 1994; 18:33-4.

[9] E Okamoto, K Tomoda, K Yamamoto, "Development of a compact, highly efficient totally implantable motor-driven assist pump system." Artificial Organs 1994; 18(12):911917.

[10] P J Ayre, N H Lovell, J C Woodard "Non-invasive flow estimation in an implantable rotary blood pump: A study considering non-pulsatile and pulsatile flows." Physiol MEA 2003; 24:179-189.

[11] Allen Cheng, Christine A. Williamitis, and Mark S. Slaughter on "Comparison on continuous flow and pulsatile flow left ventricular assist devices: is there an advantage to pulsatility" Ann Cardiothorac Surg. 2014 Nov.

[12] Puppala A. K., Sonnati, V., \& Gangapuram, S. (2020, March). "Additive Manufacturing for VADs and TAHs-a Review." In Journal of Physics: Conference Series (Vol. 1495, No. 1, p. 12021). IOP Publishing.

[13] Praveen Kumar C, Neeth Antony A on "Designing and Modelling A Ventricular Assistive Device" IOSR Journal of Electrical and Electronics Engineering, Volume 11, April 2016.

[14] S. Takatani, Kazunobu Ouchi, M. Nakamura "Miniature, Totally implantable, permanent, Electromechanical pulsatile VAD system." ASAIO J 2000 - Volume 46.

[15] William L. Holman, David C. Naftel, Chad E. Eckert, Robert L. Kormos, Daniel J. Goldstein, and James K. Kirklin, on "Durability of left ventricular assist devices: Interagency Registry for Mechanically Assisted Circulatory Support (INTERMACS)"; The Journal of Thoracic and Cardiovascular Surgery. August 2013.

[16] R. Basanth, Anil K. Puppala "Review of power delivery techniques and sources of power for VAD's and TAH's," IEEE international Conference on innovative Technologies in Engineering 2018 (ICITE OU). 